\title{
A New Methodology to Incorporate Differential Diffusion in CFD Simulations of Reactive Flows
}

\author{
G. Maragkos ${ }^{* 1}$, P. Rauwoens ${ }^{2}$, B. Merci ${ }^{1}$ \\ ${ }^{1}$ Department of Flow, Heat and Combustion Mechanics, Ghent University, Belgium \\ ${ }^{2}$ Department of Civil Engineering, Ghent University, Belgium
}

\begin{abstract}
A new methodology to take into account differential diffusion in numerical simulations of reactive flows is presented. Within this new method, the diffusion term in the transport equations of the conserved scalars consists of two parts, one expressing the diffusion between the conserved scalars and one expressing the feedback from the combustion model. The second term, which is often neglected, has been shown to have a substantial influence in the flow field. In addition, there is a reduction of the number of transport equations to be solved from the number of species (minus one) to the number of elements (minus one), and the chemical source term in the transport equations is absent. We apply the new method to a laminar, axi-symmetric $\mathrm{H}_{2} / \mathrm{N}_{2}$ - air diffusion flame and compare the calculations with experimental data. When differential diffusion effects are properly taken into account in the transport equations in physical space, simulation results agree well with the experimental data. Ignoring differential diffusion effects is not acceptable, due to lack of $\mathrm{H}_{2}$ diffusion close to the jet inlet.
\end{abstract}

\section{Introduction}

Numerical simulations of reactive flows typically involve mixtures of different chemical species, each one with different properties. Hydrogen is much lighter chemical specie when compared to other chemical components (e.g. $\mathrm{CO}_{2}, \mathrm{~N}_{2}$ or $\mathrm{O}_{2}$ ). This much smaller molecular weight of hydrogen is causing it to behave differently than the other chemical species in a mixture (to diffuse faster than other chemical species, e.g. $\mathrm{CO}_{2}$ ).

In practice, combustion processes occur in a turbulent atmosphere. Numerical simulations of turbulent combustion nowadays rely on the assumption that every chemical component diffuses the same, i.e. has the same diffusivity in the mixture. In case of fossil fuel this assumption is reasonable. In case of hydrogen combustion, however, this assumption is less valid. Mostly, this differential diffusion effect is ignored when performing numerical simulations of turbulent combustion because it either leads to great modelling simplifications or because it is expected that turbulent mixing is a far more dominant process than molecular mixing so that the turbulent diffusivity is an order of magnitude larger than the molecular diffusivity.

Apart from assuming equal mass diffusivities for all chemical species, another usual assumption made when modelling reactive flows is to consider equal thermal and mass diffusivities, leading to unity Lewis number for all chemical species. The use of these two assumptions then leads to the definition of a conserved scalar, the mixture fraction, which uniquely describes the transport of species [1]. By making use of conserved scalars (scalars whose value does not alter when they undergo a chemical reaction) the solution of the fluid movement is decoupled from the chemical reactions. This framework has formed the basis upon which many combustion models rely [2-4]. The mathematical deduction of these scalars relies on the assumption that all chemical components diffuse equally but in reality this is not the case. Yet, this is often ignored because no simple model exists that can include them or because an appropriate diffusion coefficient has to be selected for the mixture fraction in order to account for the different diffusivities of the chemical species. If differential diffusion effects are taken into account, the local species concentrations, heat release rates and flame temperatures strongly differ from the ones predicted by the equal diffusivity assumption [5]. With the new methodology presented below, differential diffusion is taken into account, while the disadvantages mentioned are avoided. It is discussed that inclusion of feedback from the combustion model is prerequisite to properly account for differential diffusion effects on transport in physical space.

\section{Description of the methodology}

Starting from the set of transport equations for chemical species, with the species mass diffusive flux expressed by Fick's law, linear combinations of the equations can be made such that the chemical source term vanishes. As such, the transport equations for the elemental mass fractions, $\eta^{\prime}=B^{\prime} Y=\left[\eta_{1}^{\prime}, \ldots, \eta_{N_{e}}^{\prime}\right]$, are obtained, written in matrix format as:

$$
\frac{\partial \rho B^{\prime} Y}{\partial t}+\nabla \cdot\left(\rho\left(\overrightarrow{u+u_{c}}\right) B^{\prime} Y\right)=\nabla \cdot\left(\rho B^{\prime} D \nabla Y\right)
$$

where $Y=\left[Y_{1}, \ldots, Y_{N_{s}}\right]$ contain the mass fraction of species $k\left(k=1, \ldots, N_{s}\right), D$ is the diffusion matrix of dimensions $N_{s} \times N_{s}, \rho$ is the density, $\vec{u}$ is the velocity vector and $B^{\prime}$ is a projection matrix of dimensions $N_{e} \times N_{s}$, projecting the full chemical space

\footnotetext{
* Corresponding author: Georgios.Maragkos@UGent.be Proceedings of the European Combustion Meeting 2013
} 
of dimensions $N_{s}$ to a subspace of conserved scalars of dimensions $N_{e}$. A correction velocity, $\vec{u}_{c}$, is also added in the convection term of the transport equations to ensure mass conservation due to the different species mass diffusion coefficients [1,6]. Other expressions can also be applied in the methodology presented below to calculate the species mass diffusion flux, e.g. the Hirschfelder-Curtiss law [6], including Soret and Dufour effects.

The proposed methodology consists of solving transport equations for all possible conserved scalars and retrieves the unresolved chemical space from a combustion model (e.g. Burke-Schumann, equilibrium chemistry or a flamelet table).

It will prove convenient to recombine the elemental mass fraction equations such that the projection matrix is orthogonal, i.e. $B B^{T}=I$, with $I$ the identity matrix. The conserved scalars are now defined as $\eta=B Y=\left[\eta_{1}, \ldots, \eta_{N_{e}}\right]$. An orthogonal matrix $U$, with dimensions $\left(N_{s}-N_{e}\right) \times N_{s}$ is also considered, projecting the full chemical space to the unresolved space, such that $U Y=C(\eta)$ can be retrieved from the combustion model. $C$ is a multidimensional function from space $N_{e}$ to space $N_{s}-N_{e}$. Because of orthogonality the property $B^{T} B+U^{T} U=I$ is verified. By introducing the above property and the conserved scalars, $\eta$, in Eqs (1):

$$
\begin{array}{r}
\frac{\partial \rho \eta}{\partial t}+\nabla \cdot\left(\rho\left(\overrightarrow{u+u_{c}}\right) \eta\right)=\nabla \cdot(\rho \underbrace{B D B^{T}}_{D_{1}} \nabla \eta) \\
+\nabla \cdot(\underbrace{\rho B D U^{T} \frac{\partial C(\eta)}{\partial \eta}}_{D_{2}} \nabla \eta)
\end{array}
$$

where $\frac{\partial C(\eta)}{\partial t}$ is the Jacobian matrix of function $C$.

In comparison to the classical mixture fraction approach, now the diffusion term of the conserved scalars consists of two parts: $D_{1}$, expressing the diffusion of the conserved scalars, and $D_{2}$, expressing the feedback from the combustion model. Note that without differential diffusion all the $N_{e}$ conserved scalars in Eqs (2) follow the same transport equation. The reader is referred to [7] for a more comprehensive presentation of the methodology.

In addition to Eqs (2), the continuity equation, the low-Mach number form of the Navier-Stokes equations and a transport equation for enthalpy are solved. The ideal gas law is used to determine the density of the mixture from the other thermodynamic variables. The transport equations for mass, momentum and enthalpy read:

$$
\begin{gathered}
\frac{\partial \rho}{\partial t}+\nabla \cdot(\rho \vec{u})=0 \\
\frac{\partial(\rho \vec{u})}{\partial t}+\nabla \cdot(\rho \vec{u} \vec{u})=-\nabla p+\nabla \cdot(\overrightarrow{\vec{\tau}})+\rho \vec{g} \\
\frac{\partial(\rho h)}{\partial t}+\nabla \cdot(\rho \vec{u} h)=\frac{\partial p}{\partial t}+\nabla \cdot(a \nabla h) \\
+\sum_{k=1}^{N_{s}}\left(\frac{1}{L e_{k}}-1\right) \nabla \cdot\left(\frac{\lambda}{c_{p}} h_{k} \nabla Y_{k}\right)+\dot{Q}
\end{gathered}
$$

where $p$ is the pressure, $\overrightarrow{\vec{\tau}}$ is the viscous stress tensor, $\vec{g}$ is the gravity vector, $h$ is the enthalpy, $\alpha$ is the thermal diffusivity and $\dot{Q}$ is the radiative heat flux. Viscous heating has been neglected in the enthalpy equation, a reasonable assumption for low-Mach number flows, while the last term accounts for the non unity Lewis number, $L e_{k} \neq 1$.

\section{Test case}

The new methodology is applied to the laminar, axisymmetric $\mathrm{H}_{2} / \mathrm{N}_{2}$ - air diffusion flames reported by Toro et al. [8]. The $\mathrm{H}_{2} / \mathrm{N}_{2}$ mixture (1:1 in mole ratio), issued with an average velocity of $0.5 \mathrm{~m} / \mathrm{s}$ from a round tube of inner diameter $\mathrm{D}=0.9 \mathrm{~cm}$, is surrounded by a co-flow of air with velocity equal to $0.5 \mathrm{~m} / \mathrm{s}$. Ambient temperature and pressure are $\mathrm{T}=298 \mathrm{~K}$ and $\mathrm{P}=101325$ $\mathrm{Pa}$, respectively, resulting in Reynolds number of $\operatorname{Re}_{D} \approx 175$.

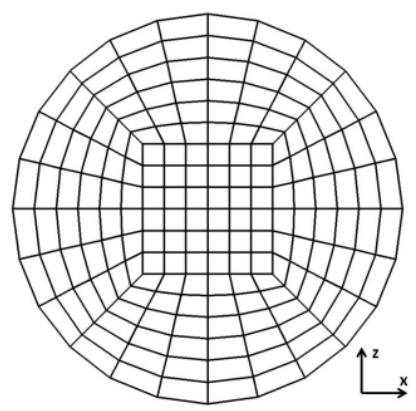

Figure 1: Schematic of mesh for fuel inlet used in the simulations.

The numerical simulations have been performed with OpenFOAM [9] with a modified version of the FireFOAM solver [10]. A cylindrical mesh is used, 10D $x$ 25D, with 18 cells across the inlet (Figure 1). Outside the inlet, 66 cells are used radially (compressed towards the inlet) and 300 cells in the axial direction. The total number of cells is then 0.562 million cells, resulting in a minimum and maximum grid spacing of $0.5 \mathrm{~mm}$ (on the centerline) and $1.84 \mathrm{~cm}$ (side planes of the domain), respectively. A parabolic profile, taken from a separate simulation with a fully developed velocity profile in a 
pipe, is used for velocity at the exit plane of the tube, similar to the one reported in the experiments. Outside the exit plane of the inlet tube, a fixed streamwise coflow velocity of $0.5 \mathrm{~m} / \mathrm{s}$ is imposed. The thickness of the tube is set to $1.0 \mathrm{~mm}$.

For this test case, $N_{s}=4$ species $k\left(\mathrm{H}_{2}, \mathrm{O}_{2}, \mathrm{H}_{2} \mathrm{O}\right.$, $\left.\mathrm{N}_{2}\right)$ and $N_{e}=3$ elements $\lambda(\mathrm{H}, \mathrm{O}, \mathrm{N})$ are considered. The species mass fractions relate to the elemental mass fractions $\eta_{H}^{\prime}$ and $\eta_{N}^{\prime}$ through the Burke - Schumann solution, as shown in Figure 2. In this case, differential diffusion effects are considered only in physical space (transport equations for the conserved scalars) and not in the combustion model (Burke - Schumann solution), but it is straightforward to apply the methodology with combustion models where differential diffusion is taken into account (e.g. in a flamelet table).

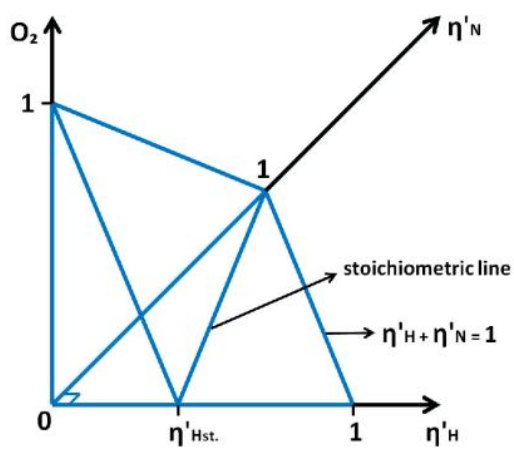

(a)

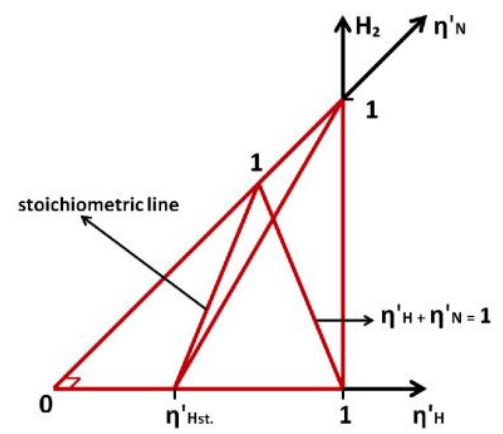

(b)

Figure 2: Burke-Schumann solution for irreversible infinitely fast chemistry, based on elemental mass fractions $\eta_{H}^{\prime}$ and $\eta_{N}^{\prime}$ for (a) $\mathrm{O}_{2}$ and (b) $\mathrm{H}_{2}$. The stoichiometric elemental mass fraction of $\mathrm{H}$ is $\eta_{H}^{\prime}=0.1119$.

The species mass diffusion coefficients, $D_{k}$, are calculated as:

$$
D_{k}=\frac{\mu}{\rho S c}
$$

assuming a constant Schmidt number for the chemical species: $S c_{\mathrm{H}_{2}}=0.21, S c_{\mathrm{O}_{2}}=0.76, S c_{\mathrm{H}_{2} \mathrm{O}}=0.63$ and $S c_{N_{2}}=0.81$ [6].
The dynamic viscosity, $\mu$, is a function of temperature and is calculated by Sutherland's law with the two Sutherland coefficients assigned the values $A_{s}=1.358519 \cdot 10^{-6}$ and $T_{s}=110.04$ for the $\mathrm{H}_{2} / \mathrm{N}_{2}$ mixture [11].

Radiation is modeled by the finite volume Discrete Ordinates Method (fvDOM), assuming that the only significant radiating species is $\mathrm{H}_{2} \mathrm{O}$ [8] .

\section{Results}

The computed temperature distributions for the cases with and without differential diffusion are presented in two-dimensional snapshots in Figure 3. The adiabatic stoichiometric temperature of the $\mathrm{H}_{2} / \mathrm{N}_{2}$ mixture is $\approx 2040 \mathrm{~K}$. The characteristic 'wishbone' flame structure [12-17] is obtained and the flame reaches its maximum temperature very close to the inlet, at the edges of the jet if differential diffusion effects are taken into account. Without differential diffusion effects, however, the maximum flame temperature, corresponds to the adiabatic flame temperature of the mixture, and is found on the centerline at a location about 10D downstream. In this case the diffusivity of $\mathrm{H}_{2}$ is about 5 times less than it should be and, as a consequence, it does not diffuse as fast towards the edge as it does in reality. As such, it is convected downstream, shifting the maximum flame temperature downstream as well.

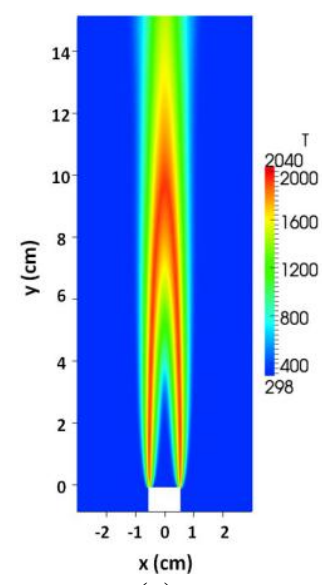

(a)

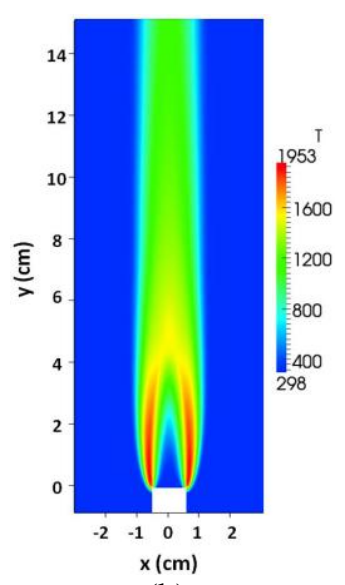

(b)
Figure 3: Two-dimensional snapshots of temperature distribution (a) without differential diffusion and (b) with differential diffusion.

Figures 4-5 present results for temperature and species $\left(\mathrm{H}_{2}, \mathrm{O}_{2}, \mathrm{H}_{2} \mathrm{O}, \mathrm{N}_{2}\right)$ mole fractions at various downstream locations. The results of the new methodology are presented with and without differential diffusion effects and compared with experimental data. The experimental data are indicated by symbols while the results with and without differential diffusion are presented with solid and dashed lines, respectively. Results by including only the $D_{1}$ diffusion term in the transport equations are also presented with dotted lines. Black symbols correspond to temperature results while 
blue, green, orange and red correspond to $\mathrm{H}_{2}, \mathrm{O}_{2}, \mathrm{~N}_{2}$ and $\mathrm{H}_{2} \mathrm{O}$ mole fraction results, respectively.

Results for temperature and species mole fractions on the centerline at location up to $\mathrm{y}=100 \mathrm{~mm}$ above the inlet are presented in Figure 4. It is observed that fuel is completely consumed by $\mathrm{y}=50 \mathrm{~mm}$, coinciding with the maximum values for temperature and $\mathrm{H}_{2} \mathrm{O}$ mole fraction. The temperature profile is well captured by the simulations with differential diffusion and the same applies for the mole fractions of species mole fractions. On the other hand if differential diffusion effects are neglected, the peak temperature on the centerline shifts much further downstream and the temperature distribution is not well captured. This also leads to discrepancies in the species mole fraction results when compared with the experimental data. Ignoring the $D_{1}$ diffusion term also leads to a strong under-prediction of the maximum flame temperature.

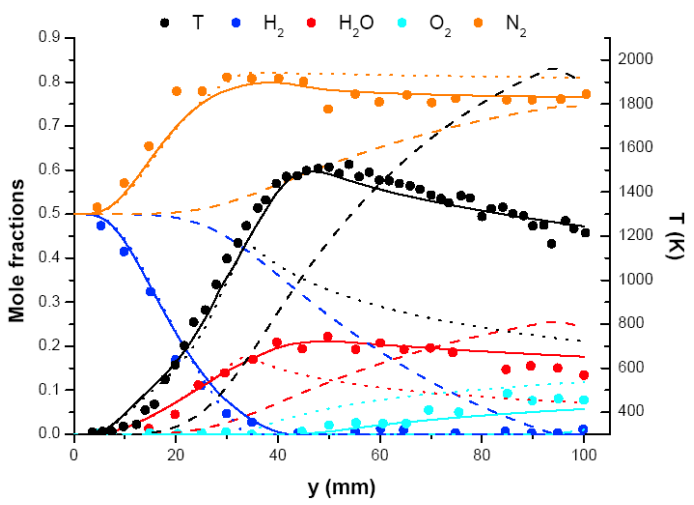

Figure 4: Temperature and species mole fractions on the centerline up to $\mathrm{y}=100 \mathrm{~mm}$. Experimental data: symbols, with differential diffusion $\left(D_{1}\right.$ and $D_{2}$ included): solid lines, with differential diffusion (only $D_{1}$ term included): dotted lines, without differential diffusion: dashed lines.

At height $y=3 \mathrm{~mm}$ (Figure 5(a)) a peak in temperature of $\mathrm{T}=1941 \mathrm{~K}$ at location $\mathrm{x}=5.8 \mathrm{~mm}$, about $1.3 \mathrm{~mm}$ outside the radius of the inlet is predicted if differential diffusion effects are considered. This is due to the high diffusivity of $\mathrm{H}_{2}$ which causes the flame to stabilize at a location outside the inlet radius. A thin zone of high temperature is observed with the peaks of $\mathrm{T}$ and $\mathrm{H}_{2} \mathrm{O}$ mole fraction, as expected, to coincide. The fuel rich (inner) and fuel lean (outer) sides of the jet are correctly predicted by the simulations and compare well with the experiments. The non-monotonic change of the mole fraction of $\mathrm{N}_{2}$, as observed by the experiments, is also observed in the simulations with differential diffusion. The peak values of $\mathrm{H}_{2}$ and $\mathrm{N}_{2}$ mole fractions are well predicted, although the profiles are a little wider than the experimental ones. Without differential diffusion the peak value of the flame temperature is similar but the lean side of the jet is under-predicted, having a much narrower profile. In this case the profiles of species mole fractions are also not well captured.

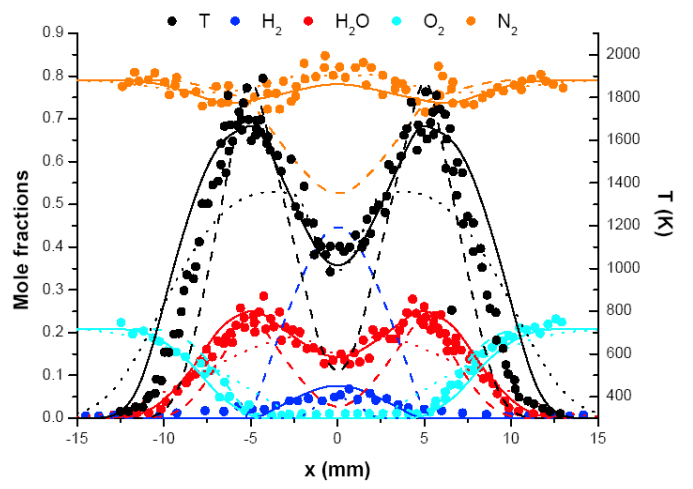

(d)

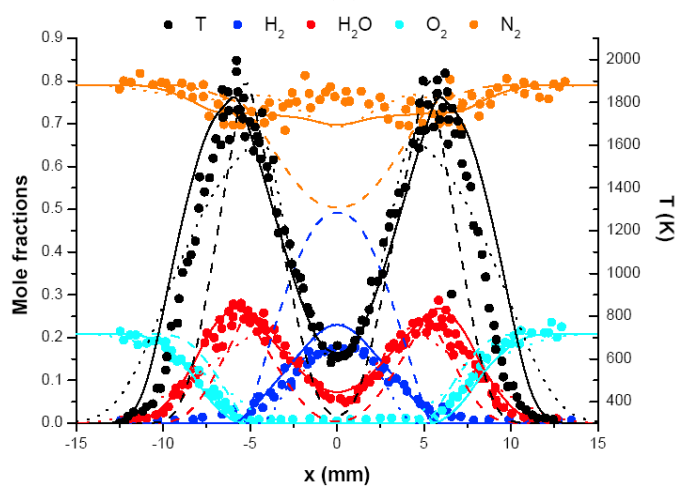

(c)

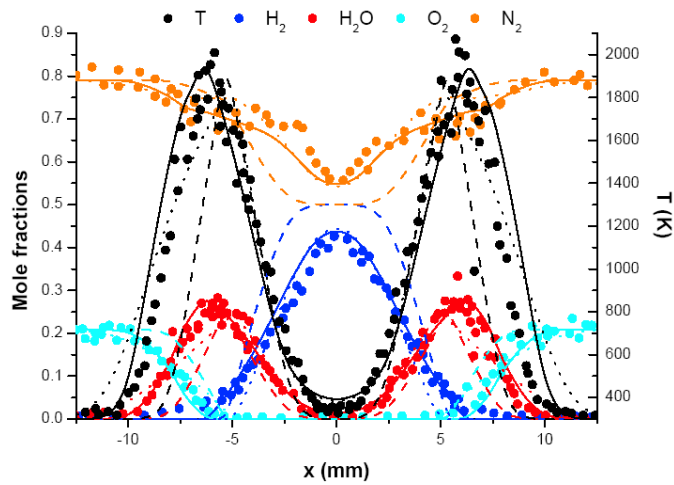

(b)

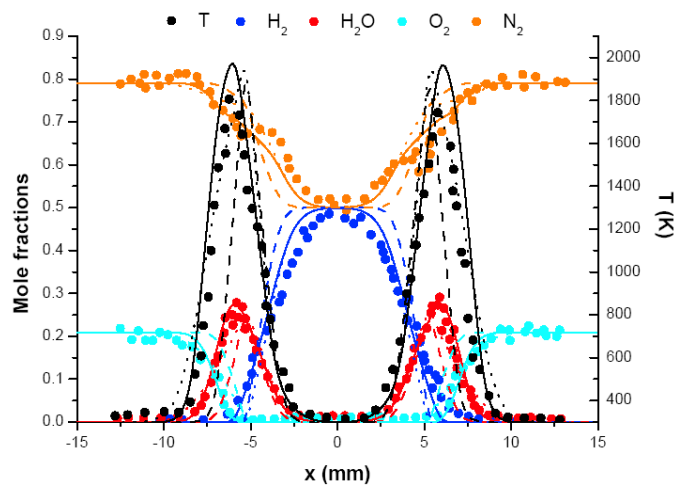

(a)

Figure 5: Temperature and species mole fractions on radial plane at height (a) $\mathrm{y}=3 \mathrm{~mm}$, (b) $\mathrm{y}=10 \mathrm{~mm}$, (c) y $=20 \mathrm{~mm}$ and (d) $\mathrm{y}=30 \mathrm{~mm}$. Experimental data: symbols, with differential diffusion $\left(D_{1}\right.$ and $D_{2}$ included): solid lines, with differential diffusion (only $D_{1}$ term included): dotted lines, without differential diffusion: dashed lines. 
At location $y=10 \mathrm{~mm}$ (Figure 5(b)), an increase in the width of the high temperature zone is observed. The peak temperature of $\mathrm{T}=1917 \mathrm{~K}$ compares quite well with the experiments. However, the lean side of the flame is slightly wider than the experiments. Even though the centerline temperature is still close to the ambient one, now $\mathrm{H}_{2}$, due to its high diffusivity, has decreased followed by an increase of $\mathrm{N}_{2}$. At this location, the non monotonic change of $\mathrm{N}_{2}$ is again quite well captured by the simulation results. The results without differential diffusion capture also quite well the peak temperature but severely under-predict the temperature at the lean side of the flame. At this location, the profiles of species mole fractions are again not well predicted.

At location $\mathrm{y}=20 \mathrm{~mm}$ (Figure 5(c)), an increase of the centerline temperature is observed, followed by a decrease of $\mathrm{H}_{2}$ and the diffusion of $\mathrm{H}_{2} \mathrm{O}$ from the reaction zone to the centerline. Here, the width of the high temperature zone has increased even more when compared to $\mathrm{y}=10 \mathrm{~mm}$. The simulations with differential diffusion effects are able to predict the temperature field and the species mole fractions quite well. At this location, the temperature profile in the lean side of the flame is again wider when compared with the experimental data. For the simulation results without differential diffusion similar observations apply like in the previous locations examined. The temperature profiles are much narrower, under-predicting the lean side of the flame.

A further increase in the centerline temperature is observed at location $\mathrm{y}=30 \mathrm{~mm}$ (Figure $5(\mathrm{~d})$ ), followed by a decrease in the mole fraction of $\mathrm{H}_{2}$. At this location, dilution with co-flow air begins to dominate the further development of the flame. Again the temperature and the species mole fractions are well captured if differential diffusion effects are taken into account. At this location the simulation results without differential diffusion don't compare well with the experiments. At much lower centerline temperature is predicted, while the $\mathrm{H}_{2}$ and $\mathrm{N}_{2}$ mole fractions are greatly over and under-predicted, respectively.

\section{Conclusions}

A new methodology has been presented to take into account differential diffusion in the transport equations in physical space. Within this new method, the diffusion term of the conserved scalars consists of two parts, one expressing the diffusion between the conserved scalars and one expressing the feedback from the combustion model. The second term, which is usually neglected, has been shown to have a substantial influence in the flow field. In addition, there is a reduction of the number of transport equations to be solved from the number of species (minus one) to the number of elements (minus one), and the chemical source term in the transport equations is absent.

Results from the application of the new model in a laminar $\mathrm{H}_{2} / \mathrm{N}_{2}$ - air diffusion flame have been presented for temperature and main species $\left(\mathrm{H}_{2}, \mathrm{H}_{2} \mathrm{O}, \mathrm{O}_{2}, \mathrm{~N}_{2}\right)$ mole fractions and compared with experimental data. If differential diffusion effects are taken into account, the comparison of the simulated results with the experimental data is very good for the temperature and species mole fractions, at all locations examined. Without differential diffusion effects, the predicted results are not in good agreement with the experiments, due to lack of $\mathrm{H}_{2}$ diffusion close to the jet inlet. This leads to a wrong prediction of the location and the peak of the flame temperature but also to a strong overprediction of the species mole fractions at all locations. Differential diffusion effects were present at the edges of the inlet, where $\mathrm{H}_{2}$ diffuses faster that the other species, but also on the centerline at locations more than 10D downstream, where there is less $\mathrm{H}_{2}$ compared to other species. The inclusion of the $D_{2}$ diffusion term has been shown to be important in order to accurately predict the temperature and species mole fractions.

While the method has been illustrated on a laminar flame, it can also be extended for turbulent flames as well. Research on this is ongoing.

\section{Acknowledgements}

This research has been funded by Ghent University (Belgium) through BOF project 01J01909.

\section{References}

[1] T. Poinsot, D. Veynante, Theoretical and Numerical combustion (First edition), Edwards, Philadelphia, USA, 2001.

[2] R.W. Bilger, Prog. Energy Combust. Sci. 1 (1976) 87-109.

[3] S.K. Liew, K.N.C. Bray, J.B. Moss, Combust. Sci. Technol. 27 (1981) 69-73.

[4] N. Peters, Proc. Combust. Inst. 21 (1988) 12311250 .

[5] L.L. Smith, R.W. Dibble, L. Talbot, R.S. Barlow, C.D. Carter, Combust. And Flame 100 (1995) 153-160.

[6] E. Giacomazzi, F.R. Picchia, N. Archidiacono, Comb. Theory Modell. 12 (2007) 135-158.

[7] G. Maragkos, P. Rauwoens, B. Merci, Combust. Flame (2013) (accepted for publication).

[8] V.V. Toro, A.V. Mokhov, H.B. Levinsky, M.D. Smooke, Proc. Combust. Inst. 30 (2005) 485-492.

[9] http://www.openfoam.com

\section{[10] http://code.google.com/p/firefoam-dev}

[11] S.C. Saxena, Proceedings of the Sixth Symposium on Thermophysical Properties, Liley, P.E,ed., American Society of Mechanical Engineers (1973) 100-110. 
[12] S.A. Northrup and C.P.T. Groth, Comp. Fluid Dyn. 4 (2009) 341-346.

[13] R.K. Mohammed, M.A. Tanoff, M.D. Smooke, A.M. Schaffer and M.B. Long, Proc. Combust. Inst. 27 (1998) 693-702.

[14] M.D. Smooke, Y. Xu, R.M. Zurn, P. Lin, J.H. Frank and M.B. Long, Proc. Combust. Inst. 24 (1992) 813-821.

[15] A.V. Mokhov, B.A.V. Bennett, H.B. Levinsky, M.D. Smooke, Proc. Combust. Inst. 31 (2007) 9971004.

[16] B.A.V. Bennett, C.S. McEnally, L.D. Pfefferle, M.D. Smooke, Combust. Flame 23 (2000) 522-546.

[17] L.L. Smith, R.W. Dibble, L. Talbot, R.S. Barlow, C.D. Carter, Combust. Flame 100 (1995) 153-160. 